\title{
Skeletal Class III malocclusion treated by pre-surgical orthodontics followed by bilateral sagittal split osteotomy
}

Hossain MZ1 BDS, PhD, Rizvi HM² BDS, Mamun MSA³ BDS and Haque S $^{4}$ BDS, MS

\begin{abstract}
Surgical correction of Dentofacial Deformities is the work of many surgeons but the orthodontist are very much involved in preparing the patients for surgery. This case report describes treatment for severe skeletal Class III with excessive reverse overjet by pre-surgical orthodontic treatment followed by bilateral sagittal split osteotomy (BSSO). The patient had a concave profile, a long lower facial height, large tongue volume and excessive mandibular protrusion and a high mandibular plane angle. The occlusion was well aligned including root paralleling in both the arches during the presurgical orthodontic treatment phase. After dental correction in both the arches, bilateral sagittal split osteotomy with mandibular push back was performed. The post treatment records showed a good facial profile and occlusion. The mandible was stable, however, there was spacing in the upper anterior segment. These results demonstrate that presurgical orthodontic treatment combined with bilateral sagittal split osteotomy resulted improvement of facial profile, aesthesis and functional need.
\end{abstract}

Keywords: Mandibular prognathism , Reverse Overjet, Pre-surgical orthodontic treatment , bilateral sagittal split osteotomy. (Ban J Orthod \& Dentofac Orthop, April 2011; Vol-1, No. 2, 9-12)

\section{INTRODUCTION}

Case Summary: The patient was a 17-year-old young girl with a chief complaint of lack of incisal contact, total crossbite, and mandibular protrusion. She had also speech problem. The patient's intelligence was very poor. Both the patient and the parents were aware of her facial look and wanted to change it by orthodontic treatment. However, during initial examination no clicking, joint pain or limitation of opening was found.

Her face in frontal view appeared long \& facial profile was concave, with chin protrusion and a long lower facial height. Tongue appeared large even in small mouth opening and there was bite mark of teeth on the dorsum of the tongue. (Fig.1: A,B,C). The patient had an Angle Class III malocclusion with total cross bite and reverse over jet (-12 $\mathrm{mm})$, reverse over bite ( $-4 \mathrm{~mm})$. and a constricted maxillary dental arch (Fig.:3: A,B,C).

Considering the above findings, this patient was diagnosed as an Angle Class III malocclusion with skeletal Class III with reverse overjet , high mandibular plane angle, and macroglossia.

\section{TREATMENT WAS PLANNED AS FOLLOWS}

Presurgical Orthodontics ${ }^{1}$ and Surgical Correction was recommended. Expansion of the maxillary dental arch with edgewise appliance, uprighting of the upper and lower molars and leveling and alignment of the upper and lower arches was considered as presurgical orthodontic treatment.

Bilateral Sagittal Split Osteotomy (BSSO) with a pushback \& fixation withTitanium Mini Plate fixation to correct the sagittal skeletal discrepancy between the maxilla and mandible and to reduce the vertical dimension of the lower facial height.1,2
Establishment of an ideal occlusal relationship through postsurgical orthodontic treatment which was not possible as patient was not available to continue post-surgical orthodontic treatment.

\section{TREATMENT PROGRESS}

Initially , edgewise appliance was placed on the upper and lower arches as presurgical orthodontic procedure (Fig.3: A,B,C). Leveling and alignment of the teeth were done including uprighting of the molars. Upper arch was expanded using nitinol arch wire. $(0.018 \times 0.025$ inch $)$. During leveling and alignment of the premolars and molars, bite opening was done by reverse curve of spee. The total presurgical orthodontic treatment time was 18 months.

After the presurgical orthodontic treatment was completed, a mandibular setback with Bilateral Sagittal Split Osteotomy1,2 (BSSO) was performed by a reputed oral \& maxillofacial surgeon. The tongue reduction was not performed, instead axial inclination of anterior teeth were over corrected. Patient was not available for Postsurgical orthodontic treatment. After debonding, a Begg-type retainer and a Hawley-type retainer was given full time on the upper and lower arches, respectively.

\section{RESULTS}

The posttreatment records showed a dramatic change in the facial profile and occlusion (Fig. 2 A,B). The protruding chin and long lower facial height were reduced, resulting in a straight profile. Acceptable overjet and overbite were was achieved. As she had missing teeth in the posterior segment, was difficult to align perfectly for anchorage control. This was compromised by surgical procedure.

\footnotetext{
${ }^{1}$ Professor \& Head, Department of Orthodontics \& Dentofacial Orthopedics, Dhaka Dental College, ${ }^{2,3}$ FCPS-II Trainee, Department of Orthodontics \& Dentofacial Orthopedics, Dhaka Dental College. ${ }^{4}$ Assistant Professor, Dept. of Conservative Dentistry, Dhaka Dental College \& Hospital
} 

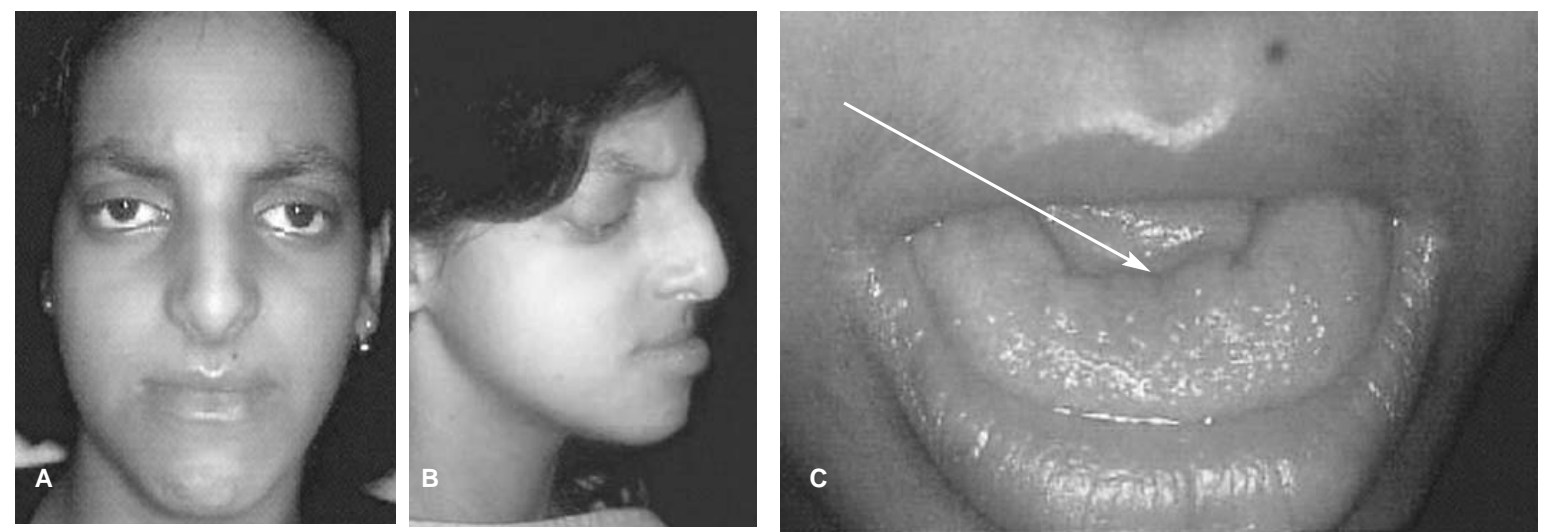

Fig.1:A,B,C Pretreatment facial photographs. : A. Pre-treatment facial photo of Severe Class III occlusion B. Pre-treatment facial profile shows severe mandibular protrusion. Note the upper \& lower lips are in imbalanced position C. Intra - oral view shows the size \& position of the tongue Larger tongue with the bite mark of the upper teeth are remarkable (Arrow Head)
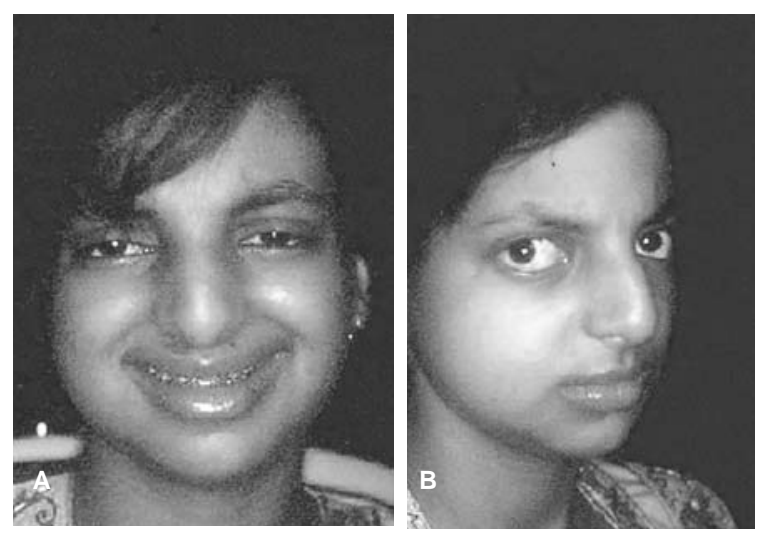

Fig. 2 : A,B (Post-surgical facial photographs) : Improved Appearance and profile after treatment. Mandible has significantly repositioned. Treatment result relieved the patient from psychological \& social embarrassment
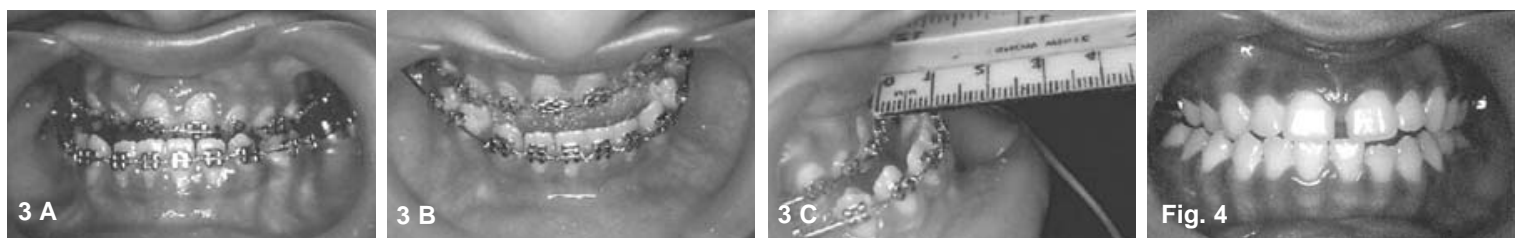

Fig. 3: A,B,C Presurgical orthodontics- intraoral photographs : Fig. 3 (A,B,C) Intra-oral view shows alignment of the occlusion as a pre-surgical orthodontic treatment $(\mathrm{A}, \mathrm{B})$. Note the degree of reverse over-jet $(-12 \mathrm{~mm})$ / maxillo-mandibular discrepancy (C)

Fig. 4: (Postsurgical orthodontics- intraoral photograph) : Just immediately after surgery photograph could not be preserved. Post surgical occlusion shows slight relapse as the patient was not so co-operative for using retainer. It was difficult to give proper interdigitation as she was reluctant to continue post surgical orthodontics.

Cephalometric evaluation ( Fig. 5 A,B,C) before and after treatment showed an acceptable repositioning of the mandible and achievement of an acceptable anteroposterior maxillomandibular relationship. Cephalometric changes were evident macroscopically.

However, scanned cephalometric radiographs (Fig.5 B,C) were screen enhanced then printed to hard copy through an Epson laser printer. The hard copy was then manually traced. ${ }^{3}$ The problems encountered include: the patient was not in centric occlusion; soft tissue out line of after treatment radiograph could not be reveled, enhancing the images might not be distortion free as each hardcopy was not up to the mark that it could be checked fully using the technique described by Baumrind. ${ }^{3}$ However tracing results revealed valuable information regarding post surgical improvement of facial aesthetics and function fig. 6 A,B. 

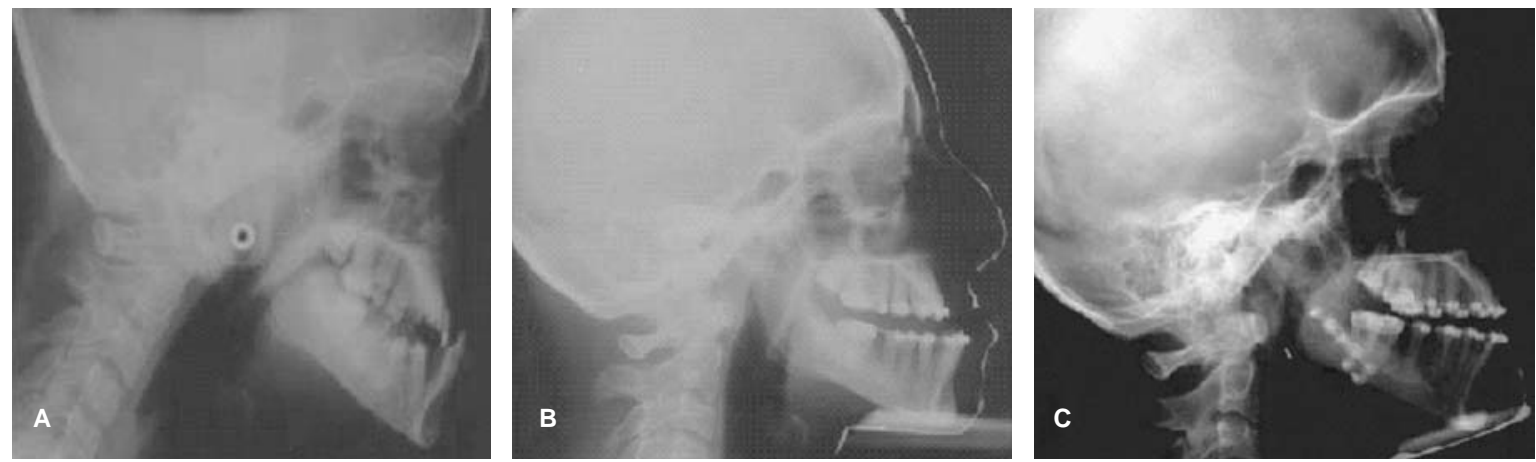

Fig. 5 : A,B,C (Cephalometric -X ray) : A. Before Orthodontic B. After Presurgical Orthodontic, crowding has been relieved C. After bilateral sagittal split osteotomy. Titanium Mini plate Fixations are seen.
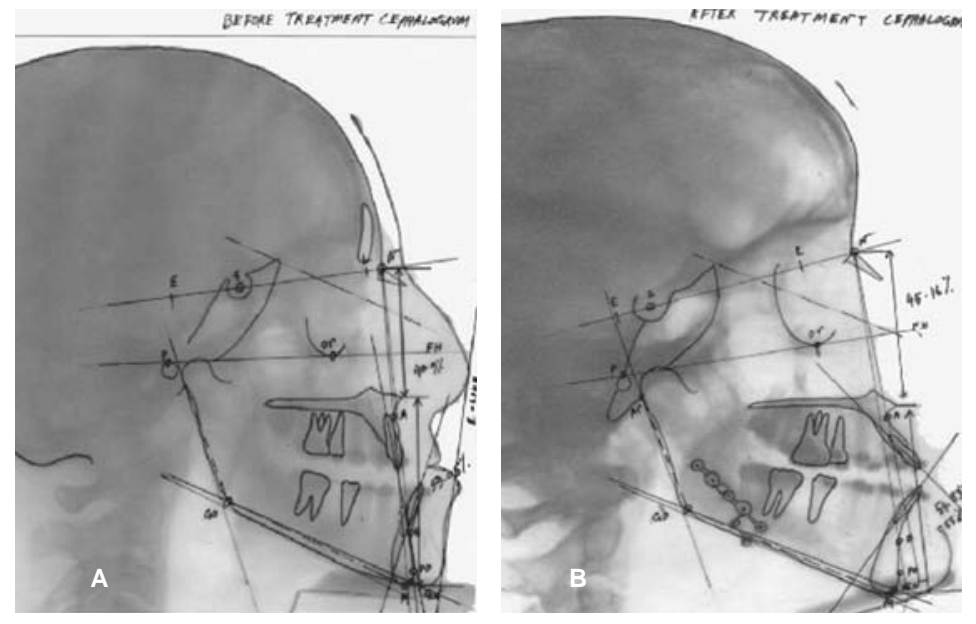

Fig. 6 :

A. Before treatment cephlogram

B. After treatment cephalogram

Table 1.1

\begin{tabular}{lll}
\hline Parameter & Before Treatment & After Treatment \\
\hline SNA (angle) & $90^{\circ}$ & $85^{\circ}$ \\
SNB (angle) & $92^{\circ}$ & $83^{\circ}$ \\
ANB (angle) & $-2^{\circ}$ & $2^{\circ}$ \\
Upper 1 to NA (mm) & $4 \mathrm{~mm}$ & $10 \mathrm{~mm}$ \\
Upper 1 to NA (angle) & $7^{\circ}$ & $33^{\circ}$ \\
Lower 1 to NB (mm) & $8.0 \mathrm{~mm}$ & $13 \mathrm{~mm}$ \\
Lower 1 to NB (angle) & $25^{\circ}$ & $42^{\circ}$ \\
Inter incisal angle (IIA) & $149^{\circ}$ & $103^{\circ}$ \\
OP to SN (angle) & Patient was not in centric occlusion & Patient was not in centric occlu- \\
& so not determined & sion so not determined \\
Go Gn to SN (angle) & Patient was not in centric occlusion & Patient was not in centric occlu- \\
& so not determined & sion so not determined \\
Gonial angle Ar-Go-Me & $136^{\circ}$ & $133^{\circ}$ \\
Lower Facial Height & $59.04 \%$ & $55 \%$ \\
E-Line & Lower lip is placed beyond this line & Soft tissue outline could not be \\
& and is protrusive & determined \\
\hline
\end{tabular}

Bangladesh Journal of Orthodontics and Dentofacial Orthopedics (BJO \& DFO) 

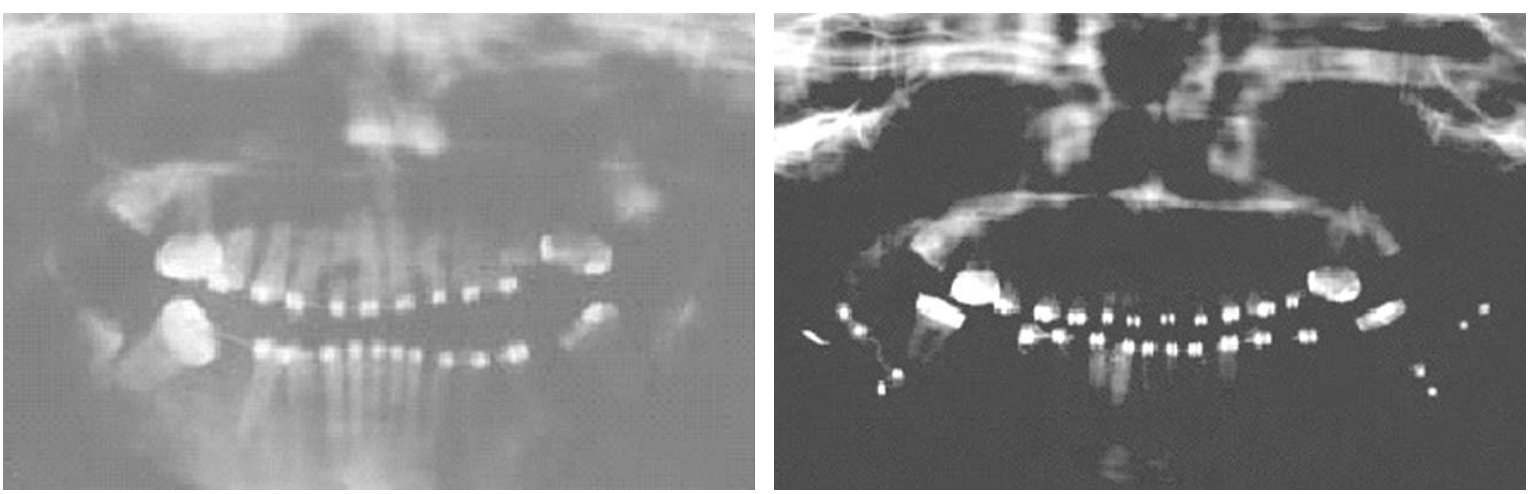

Fig. 7: A,B (OPG X-ray) :

A. Note the degree of crowding, missing lower Ist molars, mesially inclined lower right 2nd molar (Before pre-surgical orthodontics)

B. Note root paralleling after and decrowding after presurgical orthodontics followed by BSSO. Titanium mini plate fixation is visible.

Although tracing results may not be accurate due to possible distortion of image the improvement of facial esthetics table 1.1 ie establishment of a positive ANB $\left(2^{\circ}\right)$, reduction of Gonial angle toward normal $\left(133^{\circ}\right)$, decreased lower facial height towards normal(55\%) and proclination of upper lower incisors to accommodate enlarged tongue resulting acute IIA $\left(103^{\circ}\right)$ after treatment is clearly noticeable.

OPG examibnation (Fig.7 A,B) also revealed the same before presurgical orthodontics and after Surgery (BSSO).

In this case, edgewise appliance was used as a presurgical orthodontic procedure which was very essential to prepare the patient for surgical procedure and to obtain better occlusal relationship after surgical procedure. Therefore, the combination of presurgical orthodontics followed by BSSO is an an effective method for treating skeletal Class III malocclusion with mandibular prognathism.

It is well known that tongue size and position affect skeletal and dental components. ${ }^{4}$ Macroglossia has been suggested as a possible cause of mandibular prognathism, and reduction of tongue mass by partial glossectomy is an effective treatment for correcting open bite with macroglossia. ${ }^{5}$ Skeletal and soft tissue orofacial components can be changed by surgical orthodontic treatment. ${ }^{4}$ It is likely that the size of the oral cavity decreases with mandibular setback surgery and that surgery can encroach on the tongue space, even with a tongue that is of a normal size. ${ }^{6}$ The relative increase in tongue volume in the oral cavity would cause a relapse of the mandibular position after the mandibular setback, resulting in a decrease in overjet and overbite. However, tongue reduction was not performed in this case as the patient was not willing to perform the surgery. The surgeon did not consider partial glossectomy because, with the advent of the internal fixation, the surgeon did not experience relapse as much as used in the past. Moreover, the axial inclination of her anterior teeth were slight overcorrected which should take care of some of the tongue pressure.

\section{CONCLUSSION}

Presurgical orthodontic treatment followed Bilateral sagittal split osteotomy with titanium mini plate fixation can be effective for correcting the facial profile and occlusion in skeletal Class III with mandibular prognathissm.

\section{ACKNOWLEDGMENTS}

The authors would like to thank Dr. A. K Ghosh , Consultant Oral and Maxillofacial Cosmetic Surgeon, Calcutta, India for performing the BSSO surgery. Past Prssident, Association of Maxillofacial Surgeons, India.

\section{REFERENCES}

1. Bell WH, Proffit WR, White RP, Surgical Correction of Dentofacial Orthopedics, WB Saunders, 1980

2. Kharbanda OP, Orthodontics: Diagnosis and Management of of Malocclusion and Dentofacial Deformities, Elsevier, 2009, 440-449.

3. Baumrind S, Frantz RC. The reliability of head film measurements. Am J Orthod 1971;60:111-127.

4. Kawakami, M. , K. Yamamoto , T. Noshi, S. Miyawaki, and T. Kirita. Effect of surgical reduction of the tongue on dentofacial structure following mandibular setback. J Oral Maxillofac Surg 2004. 62:1188-1192.

5. Miyawaki, S. , S. Oya , H. Noguchi, et al. Long-term changes in dentoskeletal pattern in a case with Beckwith-Wiedemann syndrome following tongue reduction and orthodontic treatment. Angle Orthod 2000. 70:326-331

6. Lew, K. K. K. Changes in tongue and hyoid bone positions following anterior mandibular subapical osteotomy in patients with Class III malocclusion. Int J Adult Orthod Orthognath Surg 1993. 8:123-128

\section{Correspondence}

\section{Prof. Dr. Md. Zakir Hossain, BDS, PhD}

Prof. \& Head,

Dept. of Orthodontics \& Dentofacial Orthopedics,

Dhaka Dental College \& Hospital, Mirpur-14, Dhaka-1206.

E-mail:hossainzortho@yahoo.com,

web: www.orthodentalcare.com 